\title{
Oblique response of a split-ring-resonator-based left-handed metamaterial slab
}

\author{
Kamil Boratay Alici* and Ekmel Ozbay \\ Nanotechnology Research Center, Department of Physics, Department of Electrical and Electronics Engineering, \\ Bilkent University, Bilkent, 06800 Ankara, Turkey \\ *Corresponding author: bora@fen.bilkent.edu.tr
}

Received June 2, 2009; accepted June 25, 2009;

posted July 7, 2009 (Doc. ID 112278); published July 22, 2009

We experimentally studied the transmission response of a one-dimensional rectangle-prism-shaped metamaterial slab for oblique incidence angles. The electrical size of the split-ring resonators was 1 order of magnitude smaller than the operation wavelength $\sim 8.5 \mathrm{~cm}$. We demonstrated that the left-handed transmission peak preserved up to an angle of incidence $45^{\circ}$. The angular measurements were performed with respect to two lateral directions. The confirmed insensitivity of split-ring-resonator-based metamaterials to the angle of incidence makes them a good candidate for superlens applications. () 2009 Optical Society of America

OCIS codes: $160.3918,160.4670,260.5740$.

A narrowband negative permeability medium, which utilizes the resonant nature of the shaped metaldielectric compositions [1], was reported in the microwave regime by Smith et al. [2]. By incorporating periodically arranged thin wires [3] into this negative permeability medium they demonstrated a composite metamaterial with a negative index of refraction [1]. Thereby, the available matter-wave interactions were extended, and designs of metamaterials with a controllable effective medium response at several frequency bands of electromagnetic spectrum were realized [4-9].

Metal-dielectric-based metamaterials are demonstrated to operate for normal incidence at around $5 \mathrm{GHz}$ [1], $10 \mathrm{GHz}$ [4,5], $100 \mathrm{GHz}$ [6], $5 \mathrm{THz}$ [7], $100 \mathrm{THz}$ [8], and $370 \mathrm{THz}$ [9] by scaling the physical size of the constituting resonators and wires. However, owing to the ohmic losses the magnetic resonance of the elements saturate, it is rather difficult to verify the negative index concepts at the visible regime [10]. Moreover, the fabrication of such metallic shapes that have linewidths of several tens of nanometers is a challenging task. Some recent designs increased the operation frequency but at the expense of effective medium concepts. They used elements of electrical size that are comparable to the operation wavelength [11]. To be able apply the effective medium characterization methods to the designed metamaterials, several points should be kept in mind. First, the electrical size of the resonators are to be at least 1 order of magnitude smaller than the operation wavelength. Second, the number of metamaterial layers at the propagation direction should be large enough that the parameters permeability and permittivity are meaningful. Third, the metamaterial should have an invariant response for oblique incidence, especially for the exciting applications such as subwavelength imaging and the focusing of light.

In the present Letter, we focus on the response of a split-ring-resonator (SRR)-based composite metamaterial. The results are presented at the $S$ band for the case when the incident beam has a nonzero incidence angle. First, we introduce our design and structure parameters, and then we show the results of the qualitative effective medium theory (QEMT) characterization method. The frequency dependent response of a three-layered composite metamaterial, which was illuminated with an oblique angle with respect to two bases, will be shown experimentally.

The studies for the characterization of metamaterials by using scattering parameters started just after the first experimental demonstration [2]. There are two well-defined methods to determine whether a metamaterial slab is of negative index from the $S$ parameters: (i) the standard retrieval analysis $[12,13]$ and (ii) the transmission-based QEMT [14]. For the standard retrieval analysis, normal incidence complex transmission and the reflection coefficients of a metamaterial slab need to be measured precisely. Then, by comparing the complex $S$ parameters of this metamaterial medium with $S$ parameters of a homogeneous slab, an effective index $(n)$ and impedance (z) can be assigned to the metamaterial medium. Careful implementation of this method yields index, impedance, and thereby permittivity $(\varepsilon=n z)$ and permeability $(\mu=n / z)$ at the frequency band of interest. Retrieval analysis is rather difficult compared with the QEMT, in which only the transmission measurements are necessary. For this characterization method at least four different structures need to be fabricated: (i) negative permeability medium composed of electrically small resonators; (ii) doublenegative medium with incorporated continuous wires; and (iii), (iv) their shorted versions. From the transmission response of these four structures, the double-negative nature of the metamaterial can be determined.

The electrically small resonator part of the designed unit cell is shown in Fig. 1. At the microwave frequencies, we prefer to utilize the printed circuitboard technology for the fabrication of the metamaterial samples. By etching the metal-coated substrates as desired, SRR shapes were obtained with following parameters: the strip width, $w=0.9 \mathrm{~mm}$; the separa- 


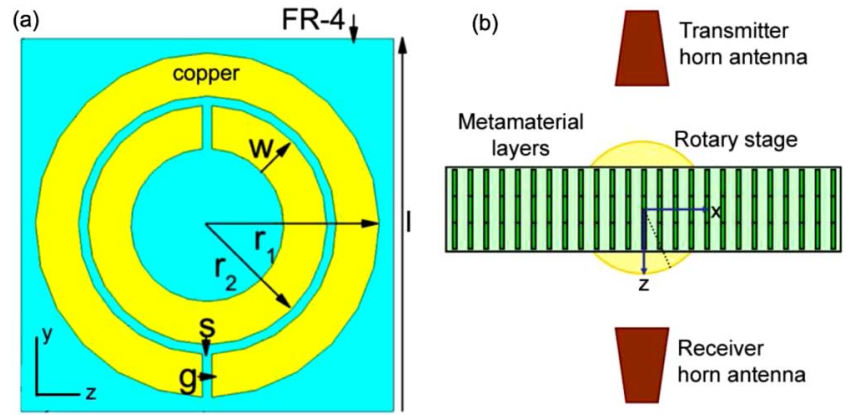

Fig. 1. (Color online) (a) Unit cell parameters. (b) Schematic of the experiment setup when the metamaterial slab was rotated with respect to the $y$ direction.

tion between the adjacent strips, $s=0.2 \mathrm{~mm}$; the split width, $g=0.2 \mathrm{~mm}$; the outer ring radius, $r_{1}=3.6 \mathrm{~mm}$; and the inner ring radius, $r_{2}=2.5 \mathrm{~mm}$. The period of the resonators at the $y$ and $z$ directions was $l$ $=8.8 \mathrm{~mm}$. The substrate was FR-4 with relative permittivity, $\varepsilon=3.75$, and the dissipation factor, $\tan \delta$ $=0.002$. The thickness of the substrate was $1.6 \mathrm{~mm}$, and the deposited copper thickness was $30 \mu \mathrm{m}$. On the back side of the substrate, centered continuous wires were fabricated with a width of $w=0.9 \mathrm{~mm}$.

The QEMT analysis was performed numerically by using CST-MWS. The unit cell of the medium was illuminated by a normally incident plane wave, and the transmitted signal was obtained via point field probes. The SRR medium transmission spectra had a stop band at around the resonance frequency. When we measured the transmission response of its shorted version (sh-SRR) the stop band disappeared, demonstrating the fact that the resonance was magnetically originated. For the sh-SRR, the split width is zero $(g=0)$, and thereby the capacitance of the SRR is shorted. We conclude this part of the QEMT by stating that at around the magnetic resonance frequency, the medium has negative effective permeability $(\mu<0)$. In the second part, we incorporate continuous wires and simulate the transmission response of the composite metamaterial (CMM). In this case, we see a transmission band at around the resonance frequency and inspect its double-negative origin. If it was so, the transmission band needed to disappear for the sh-CMM case, in which we had shSRRs instead of SRRs and the permeability was not negative. As can be seen in Fig. 2, below the plasma frequency of the sh-CMM medium there was no transmission band, which implies the double negativity of the designed CMM metamaterial. In general, the QEMT method is preferred if the fabrication of the four different media can be processed easily.

As we have in fact confirmed that our metamaterial has a negative index of refraction $(n<0)$ at the double-negative transmission band, we can start demonstrating the results of the oblique illumination study. In Fig. 1(b) a schematic of the experiment setup for the rotation with respect to the $y$ direction is shown. Standard microwave horn antennas were used as the source and sink of the radiation. We measured the transmission response for a spectrum of incidence angles by using an N5230A Vector Network

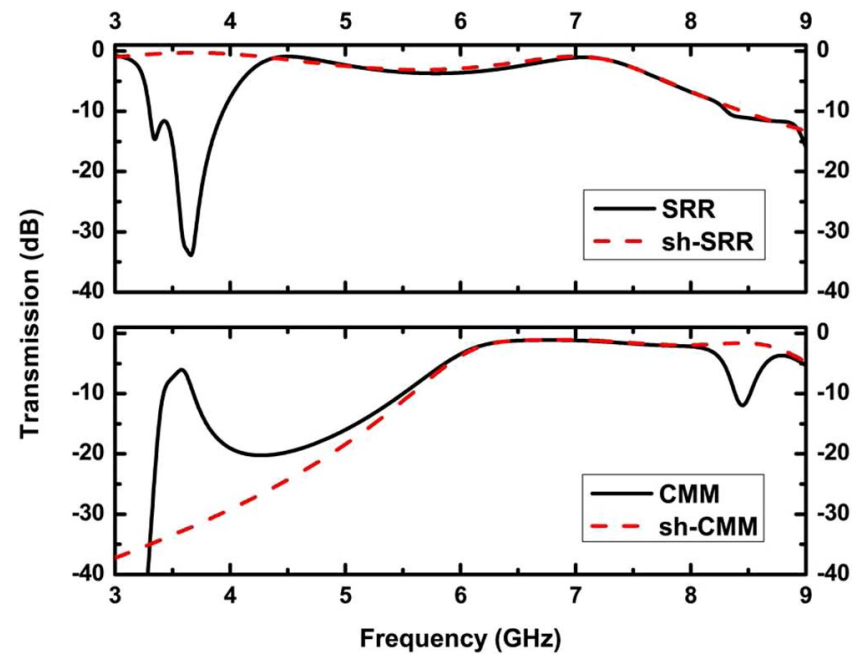

Fig. 2. (Color online) Results of the QEMT. Transmission response of the SRR medium and its shorted version (sh$\mathrm{SRR}$ ), and the CMM and its shorted version (sh-CMM) are shown.

Analyzer and a computer-controlled rotary stage. We have shown the results of two different measurements: (i) a CMM slab of three layers at the propagation direction, which was rotated with respect to the $y$-axis with an angle $\theta$ ranging from $0^{\circ}$ to $45^{\circ}$, and (ii)

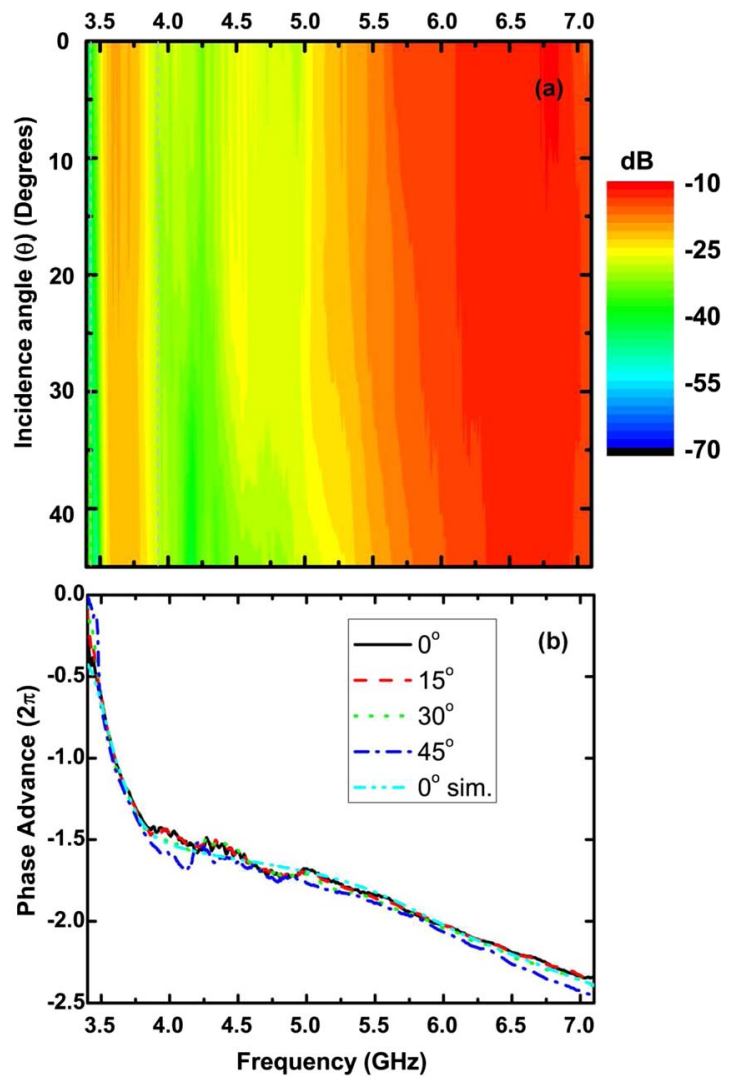

Fig. 3. (Color online) (a) Experimental transmission spectra as a function of frequency and angle of incidence $\theta$ for the three-layered composite metamaterial are shown. The angle $\theta$ corresponds to rotation with respect to the $y$ axis. (b) Experimental transmission phase data for selected incidence angles $0^{\circ}, 15^{\circ}, 30^{\circ}, 45^{\circ}$ and corresponding simulation for $0^{\circ}$ incidence angle. 
the three-layered CMM that was rotated with respect to the $x$ axis with an angle $\alpha$ ranging from $0^{\circ}$ to $45^{\circ}$.

Figure 3(a) shows that as the angle of incidence increased from $0^{\circ}$ to $45^{\circ}$, the negative transmission band remained with only a minor change: peak transmission value had changed on the order of 1 or $2 \mathrm{~dB}$. Moreover, as the angle of incidence increased a narrower and slightly shifted double-negative band was observed. Its response remained nearly the same at a considerable fraction of the frequency band. In Fig. 3(b) we plotted the experimental transmission phase advance for selected incidence angles: $0^{\circ}, 15^{\circ}$, $30^{\circ}, 45^{\circ}$, and related simulation for $0^{\circ}$. At the negative band, as the incidence angle increased the behavior of the transmission phase remained almost the same, in good agreement with the simulation. In Fig. 4(a), we show the results for the three-layered case when the incidence angle was varied with respect to the $x$ axis. The change of the response at the frequency band and angular domain are minor. In this case, the SRRs were excited both magnetically and electrically; therefore, we saw a strengthened transmission response as the angle $\alpha$ increased. At this point, we would like to clarify that the transmission peak for this oblique incidence is indeed due to double-negative nature. When the incidence angle with respect to the $x$ axis was $45^{\circ}$, we simulated the



Fig. 4. (Color online) (a) Transmission spectra as a function of the frequency and angle of incidence $\alpha$ for the threelayered composite metamaterial are shown. The angle $\alpha$ corresponds to rotation with respect to the $x$ axis. (b) Simulated transmission response of a semi-infinite continuous wire array and CCMM for the incidence angle of $45^{\circ}$. response of finite length continuous wire array and the sh-CMM. There were $20 x$-axis unit cells, infinitely periodic $y$-axis unit cells, and three unit cells at the propagation direction. In Fig. 4(b) we show that the plasma frequency of the finite-length shCMM medium is around 6.5 GHz. Thus our metamaterial has negative permittivity for the oblique incidence with respect to the $x$ axis below $6.5 \mathrm{GHz}$. As we have a transmission band and negative permittivity at around the resonance frequency in Fig. 4, the medium's permeability has to be negative, as expected.

In summary, we systematically studied a doublenegative three-layered metamaterial slab oblique response and showed that the negative index characteristics remain nearly the same up to incidence angle of $45^{\circ}$. The negative transmission band remained almost the same for two different bases of rotation. The insensitivity of SRR-based metamaterials to the angle of incidence makes them a good candidate for metamaterial applications, especially the superlens.

This work is supported by the European Union under the projects EU-METAMORPHOSE, EUPHOREMOST, EU-PHOME, and EU-ECONAM, and TUBITAK under the project numbers 105E066, 105A005, 106E198, and 106A017. One of the authors (E. O.) also acknowledges partial support from the Turkish Academy of Sciences.

\section{References}

1. J. B. Pendry, A. J. Holden, D. J. Robbins, and W. J. Stewart, IEEE Trans. Microwave Theory Tech. 47, 2075 (1999).

2. D. R. Smith, W. J. Padilla, D. C. Vier, S. C. NematNasser, and S. Schultz, Phys. Rev. Lett. 84, 4184 (2000).

3. J. B. Pendry, A. J. Holden, W. J. Stewart, and I. Youngs, Phys. Rev. Lett. 76, 4773 (1996).

4. R. A. Shelby, D. R. Smith, and S. Schultz, Science 292 , 77 (2001).

5. K. B. Alici and E. Ozbay, Photon. Nanostruct. 6, 102 (2008).

6. K. B. Alici and E. Ozbay, J. Phys. D 41, 135011 (2008).

7. B. D. F. Casse, M. O. Moser, J. W. Lee, M. Bahou, S. Inglis, and L. K. Jian, Appl. Phys. Lett. 90, 254106(1) (2007).

8. S. Linden, C. Enkrich, M. Wegener, J. Zhou, Th. Koschny, and C. M. Soukoulis, Science 306, 1351 (2004).

9. C. Enkrich, M. Wegener, S. Linden, S. Burger, L. Zschiedrich, F. Schmidt, J. F. Zhou, Th. Koschny, and C. M. Soukoulis, Phys. Rev. Lett. 95, 203901(1) (2005).

10. J. Zhou, Th. Koschny, M. Kafesaki, E. N. Economou, J. B. Pendry, and C. M. Soukoulis, Phys. Rev. Lett. 95, 223902(1) (2005).

11. G. Dolling, M. Wegener, C. M. Soukoulis, and S. Linden, Opt. Lett. 32, 53 (2007).

12. D. R. Smith, S. Shultz, P. Markos, and C. M. Soukoulis, Phys. Rev. B 65, 195104(1) (2002).

13. X. Chen, T. M. Grzegorczyk, B. I. Wu, J. Pacheco, and J. A. Kong, Phys. Rev. E 70, 016608(1) (2004).

14. Th. Koschny, M. Kafesaki, E. N. Economou, and C. M. Soukoulis, Phys. Rev. Lett. 93, 107402(1) (2004). 